\title{
Improving the method of assessing road safety at intersections of single-level highways
}

\author{
Botir Abdullaev*, Davron Yuldoshev, Tolqin Muminov, and Dilmurod Axmedov \\ Tashkent State Transport University, Tashkent, Uzbekistan
}

\begin{abstract}
The issues of public transport service, its quality, road safety along the route are becoming a priority worldwide. After all, ensuring the safety of public transport serves to increase its attractiveness. Researchbased on different approaches to road safety is being conducted in the areas. However, the issue of road safety in public transport remains relevant. Traffic accidents on bus routes occur mainly at intersections, haul sections, and intermediate stops. Intersections of single-level highways are among the areas where traffic accidents are most likely to occur. It should be noted that the assessment of road safety in public transport is based not only on traffic accidents and violations but also on the intensity and variability of traffic and pedestrian traffic, the degree of organization of traffic, road condition and geometric elements, parameters of intermediate stations and many other factors. It is advisable to tie. Therefore, this research work aims to assess the traffic safety of directional buses and consists of several stages. This article is devoted to improving the method of assessing road safety at intersections of single-level highways.
\end{abstract}

\section{Introduction}

Most passenger cars have a capacity of up to four passengers, and in very few cases, full capacity is used. Currently, the capacity of MAN buses in Tashkent is 90, Mercedes Bents Low Floor - 110 passengers, and in "rush hour," in most cases, the full capacity is used. Transport services for the main part of passengers are provided by bus routes of JSC "Toshshahartransxizmat." Annually about 240 million passengers are transported to their destinations. This, in turn, necessitates scientific research on road safety on the routes.

Population growth and the number of cars in the country will lead to an increase in traffic. This, in turn, leads to increased levels of traffic congestion, traffic collision (TC), noise, and pollution of the environment with toxic gases. Such cases are becoming a global problem, especially in large cities. All this increases the demand for the quality of public transport services.

The level of passenger safety in public transport is one indicator of service quality [1, 2]. Improving the quality of public transport due to road safety is becoming a priority. There is a lot of scientific research being done in this regard by researchers around the world. For example, on issues of traffic safety at bus stops, including factors leading to the death of passengers to the parameters of their movement at the station [3], development of a

*Corresponding author: mrabotir@maile.ru 
methodology for assessing the safety of bus stops [4], optimal design of bus stops, the relationship between passengers and bus traffic, studies of bus delays and their effect on capacity [5]. Also, research was carried out on factors affecting road safety on routes, intersections, and crossings and an assessment of the risk of traffic accidents. In particular, researchers from the Department of Civil and Environmental Engineering of School of Engineering University of Cataina Salvatore Cafiso, Alessandro Di Graziano, Giuseppina Pappalardolar conducted a five-stage problem analysis survey among bus drivers and passengers. Kendall investigated the relationship between driver skills, age, fatigue rate, work experience, and bus safety based on compliance rate results [6].

A new method of assessing the risk of traffic accidents on bus routes has been developed by researchers Fabio Porcu, Alessandro Olivo, Giulio Maternini, Benedetto Barabino. The method is based on identifying risk components based on traffic frequency and factors that can cause bus accidents. When creating a new route based on identified and collected data, the rating of safety indicators for each route is determined by the function "Dent" [7].

Syyed Adnan Raheel Shah, a researcher at the Taxila Institute of Transportation Engineering, Department of Civil Engineering, and others, have studied the link between road safety and the factors that affect it. In the first phase of the study, the DEA method was used to calculate and sort road safety in Asian countries. In the second step, a systematic equation model (SEM) with hidden variable indicators was used, and indicators of road hazard levels and the interaction of hidden variables were analyzed [8].

I Farida's study was devoted to the safety of public transport services and conducted questionnaires and interviews on 332 public transport services. The survey found that $40.1 \%$ of the services provided were in "average" level, in addition to lack of comfort and safety factors, overcrowded buses, lack of regularity of routes (Not completing the last bus trips: due to a small number of passengers or at the discretion of the driver) and poor driver behavior. It has also been found that some drivers neglect safety and comfort. [9].

Huaguo, Zhou, Pei-Sung Lin, Joan Shen researched unconventional design for buses, safe turning at signaled intersections. The study analyzed the level of collision of buses at intersections, the number of conflict situations. Rates have been developed at intersections to analyze conflicts. Factors influencing the turn of buses at intersections and the relationship between the intersection and the buses and critical safety levels were calculated [10].

Anna Isabel J. Toostes, Fatima de L.P. Duarte-Figueiredo, Renato Assuncao, Juliana Salles, Antonio AF Loureiro studied traffic congestion on the streets of Chicago using geographic information systems in their studies and analyzed the regression of the results. 98\% accuracy was obtained from the use of geographic information systems [11].

Amit Prasad Timalsena, Anil Marsani, Hemant Tiwari studied congestion on the Maitighar-Baneshwor-Tinkune highway (morning and evening rush hours) and analyzed the time lost by passengers by mode of transport (bicycles, cars, buses of different capacities). It was found that a total of 6464.90 hours were lost on Maitighar Street, and the annual loss was 211.81 years. Only on weekdays (excluding Saturdays and holidays) and on two types of buses (Bus and Standard Bus), a loss of $70 \%$ of the time passengers spend on travel has been identified [12]. To reduce the time spent by passengers, it was decided to prioritize the traffic on the route "Tinkune - Maitighar" in the morning "rush hour" and in the evening "rush hour" on the route "Maitighar-Tinkune."

Alexandra Koźlak, Dagmara Wachlar conducted a study on the factors influencing traffic congestion in major Polish cities. The number of businesses and cars in the city had the greatest impact on congestion in Polish cities, with the least impact on the number of roads per 10,000 people and transport and communication costs per person [13]. 
In the study of Mrs.Swati Chandurkar, Sneha Mugade, Sanjana Sinha, Megharani Example, Pooja Borekar, passengers can get real-time information about RTPIS (Real Time Passenger Information) system in case of bus delays, unexpected delays, irregular routes, and other possibility cases[14].

Seiya Matsuoka is a research project on the installation of modern traffic signs, 196 vehicle video detectors, and 26 surveillance video cameras at 109 intersections on Phnom Penh Street in Japan and the resulting efficiency (traffic time, fuel consumption, emissions, number of traffic jams, traffic congestion, reducing stops, increasing average speed). At the object of study, the hourly traffic intensity (in both directions) was increased by $2.3 \%$, and the total length of the queue was reduced by $12.3 \%$ [15].

Analysis of the research showed that a lot of research had been done on road safety in some parts of public transport routes, such as road crossings, intermediate stations, or haul sections. Still, throughout the route (road crossings, intermediate stations, haul sections) insufficient research has been conducted on road safety.

The study aims to develop methods for assessing the safety of directional buses at single-level intersections, haul sections, and intermediate stops to improve existing methods. The research involves several stages. This article is aimed at improving the method of assessing road safety at single-level intersections.

\section{Research and methods}

Researchers around the world have different views on evaluating the quality of public transport services. For example, Ostrovsky's N B criterion considers the use of the vehicle's capacity, the relativity of the time spent by passengers, the regularity of the movement, and the coefficients of variation of road accidents [16]. Kravchenko's criteria EA takes into account the relative congestion of rolling stock, departures, regularity of traffic, the density of transport networks, transfer of passengers to another mode of transport, coefficient of use by distance, coefficient of uneven passenger traffic, coefficients of dynamic changes in the level of traffic, technical, operational and permissible speeds [18]. According to these criteria, public transport safety is assessed by the coefficient of variation of traffic accidents. The coefficient of variation of road traffic accidents (RTA) considers the coefficient of relative loss of public transport passengers as a result of RTAs and the dynamic level of RTA [17, 18]. The dynamic rate of RTA is related to the number of penalty points because of RTA and traffic violations, as well as the distance traveled by bus in penalty points [19].

A perfect result cannot be obtained by assessing the safety of public transport traffic with the coefficient of variation of traffic accidents. This is because the coefficient of variation of traffic collisions (TC) remains related to the consequences of TC and the driver's violation of traffic rules. The criteria mentioned above cannot be used to assess traffic safety on rerouted or newly opened routes. Also, traffic safety on different routes with the same value of the parameters that make up the coefficient of variation of RTA over a given period can not be considered the same. Perhaps public transport safety is more related to the complexity of traffic conditions along the route, such as intersections, crossings, their number, traffic organization, traffic volume, composition, conflict points, and their hazard levels. The probability of TCs also depends on these factors. Therefore, it is necessary to improve the existing criteria for assessing the safety of public transport.

When assessing public transport safety, the route should be divided into 3 parts: intersections, crossings, and stations.

Each section is further subdivided into groups, e.g.

1. Intersections

1.1. highway - highway; 
1.2. highway - railway;

1.3. highway - waterway, etc.

Small groups should also be divided into groups, for example, intersections:

1.1.1. one-level intersection;

1.1.2. multi-level intersection, etc.

These groups are subdivided into smaller groups, such as single-level intersections:

1.1.1.1. managed intersections;

1.1.1.2. uncontrolled intersections, etc.

Subgroups continue to be further subdivided into subgroups.

The rest of the same line should be divided into groups. Then it is possible to determine the coefficient of traffic safety on the route by the following expression,

$$
K_{x_{y \mu}}=\prod_{i=1}^{n} K_{i}
$$

where: Ki are aggregated safety coefficients at intersections, hauls, and stations.

$\mathrm{Ki}$ is calculated separately for each object and forms the total coefficient of the respective objects; for example, the final traffic safety coefficient of the total intersections in the direction is equal to.

$$
K_{\kappa_{y n}}=\prod_{i=1}^{n} K_{j}
$$

where: $\mathrm{Kj}$ are traffic safety coefficients at the intersections.

The calculation of the traffic safety coefficient for all groups will continue in the same way.

Traffic safety at a single-level intersection of a highway with a highway depends on the intensity of traffic and pedestrian flows crossing it, its unevenness coefficient, conflict points, and their relative destructions.

The danger of conflicting points can be assessed by its destructiveness [20]. The level of danger at an intersection is characterized by the number of accidents in which 10 million vehicles pass [19]. This method of assessing road safety at single-level intersections of highways does not take into account pedestrian traffic. However, at intersections of highways, traffic accidents can also occur at intersections of vehicles and pedestrians. As a result of the research, the safety of single-level intersections of highways is determined by: the theoretical probabilities of the occurrence of traffic accidents at the intersection of vehicles and at the intersection of vehicles and pedestrians; annual coefficients of uneven traffic of vehicles and pedestrians; an expression was developed relating to the intensity of traffic on the main and secondary roads and the intensity of pedestrian traffic at the entrance and exit of the intersection.

Indicators other than the theoretical probability of RTAs at points of conflict between vehicles and pedestrians can be determined through testing in an expression designed to assess the safety of road crossings. Determining the theoretical probability of a traffic accident at a point of conflict between vehicles and pedestrians is a bit complicated. It depends on the theoretical probability of a traffic accident at a conflict point at a given time and the number of conflict points the intersection.

The theoretical probability of an accident in a given period at conflicting points of traffic flows depends on the angles at the points of intersection of traffic flows, radii at the points of docking, and separation. Below is the determination of the angles at the points of intersection of traffic flows. 


\section{Methods}

Road traffic accidents can occur at any intersection where traffic intersects with each other or with pedestrian traffic. Therefore, test studies can be conducted at any intersection where public transport operates. The object of the study was the intersection of the streets of Tashkent (single-level) "Small Ring Road - Bogibuston" with four and two lanes.

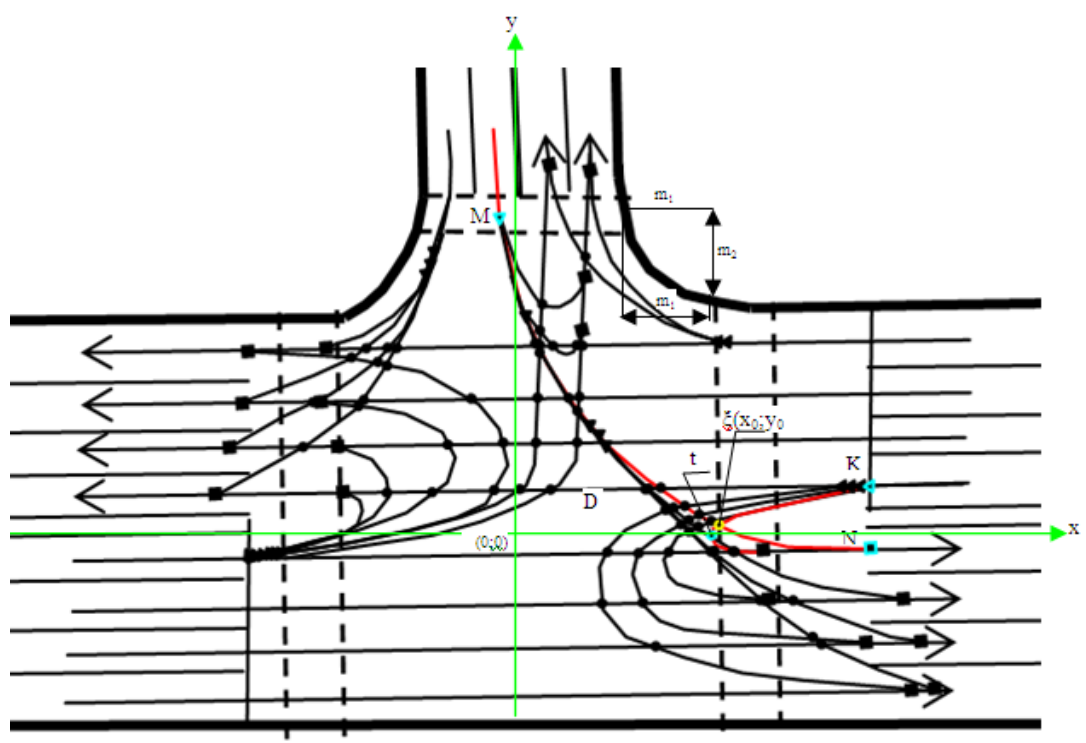

Fig.1. The scheme of the intersection of the streets of Tashkent "Small ring highway - Bogibuston."

To determine the angles at the points of intersection of traffic flows, it is first necessary to prepare a schematic view of the intersection of highways and the trajectories of traffic flows. A schematic view of the intersection was drawn using the passport of the roads, Google Maps, Google Earth. The traffic flow in it was processed by mathematicalstatistical methods to ensure sufficient reliability of the samples taken along the routes. The most repetitive variant of the trajectories of right, left, right, and reverse turns was selected (Figure 1). The points of intersection $(\bullet)$, separation $(\Delta)$, and addition $(\square)$ of the traffic flows in which RTAs can occur have been identified.

\section{Results and Discussion}

To determine the angles at the points of intersection of traffic flows, the center of the intersection of highways was placed at the point of the coordinate system ( $0 ; 0)$ (Fig. 1). The point of intersection formed by the intersection of the trajectories of reversing and left turns of vehicles is considered to be the point of intersection of the lines of the hyperbola and parabola, respectively, using the following parabola and hyperbola equations (3) and (4):

$$
\begin{aligned}
& y=a x^{2}+b x+c \\
& y=\frac{k}{x-x_{0}}+y_{0}
\end{aligned}
$$


The car moves in a straight line until it reaches and exits the intersection and in a curved line inside the intersection. Points "M" and "N," which change from rectilinear to curved and again from rectilinear to rectilinear; pivot point " $\mathrm{k}$ "; the turning point, that is, the end of the parabola, was defined as "D," and the distance from this point to the border of the intersection (to the point at the intersection of the carriageway) was defined as $\mathrm{m} 2$ and $\mathrm{m} 1$.

The coordinates of the points "M" and "N" were determined by marking the width of the carriageway on the ordinate and abscissa axes as "a" and "b," respectively.

The resulting parabola and hyperbola equations are then derived,

$$
\left\{\begin{array}{l}
K_{1}=y_{g}{ }^{\prime}\left(x_{0}\right) \\
K_{2}=y_{p}^{\prime}\left(x_{0}\right)
\end{array}\right.
$$

The angle between the points where the lines of the parabola and the hyperbola intersect is determined by the following expression:

$$
\alpha=\operatorname{arctg}\left|\frac{K_{1}-K_{2}}{1+K_{1} K_{2}}\right|
$$

According to expression (6), at the point "t," the direction of traffic flow intersects at an angle of $112^{\circ}$.

After determining the angles at the points of intersection of traffic flows, radii at the points of separation and joining, it will be possible to assess the level of danger at the intersection. In this case, all types of vehicles are considered passenger car units. Further research will focus on developing proposals to improve safety at single-level intersections of highways based on the results of the calculation and the improvement of methods for assessing road safety at intermediate stops and haul sections of bus routes.

\section{Conclusions}

1. As an object of research, an option was selected that provides sufficient reliability of the trajectories of traffic flows along the routes.

2. At the research object, the angle of intersection of the trajectory of the traffic flow to the left and back was determined.

3. The existing methodology for assessing road safety at single-level intersections of highways has been improved. 


\section{References}

1. EN 13816:2002. Transportation - Logistics and Services - Public Passenger Transport - Service Quality Definition, Targeting and Measurement. European Committee for Standardization, (2002).

2. State standard GOST R 51004 - 96. Transport services. Passenger Transportation. Nomenclature of quality indicators, - M .: IPK Publishing house of standards, $\mathrm{p} 9$. (1997).

3. Sneha Lakhotia, Sylvain Lassarre, K. Ramachandra Rao, Geetam Tiwari, Pedestrian accessibility and safety around bus stops in Delhi, S. Lakhotia et al. / IATSS Research (44) pp. 55-66. (2020),

4. Munavar Fairooz Cheranchery, Kinjal Bhattacharyya, Bhargab Maitra, Manfred Boltze., Assessing Safety Level of Bus Stops in the Absence of Crash Data, pp. 16. (2016).

5. K.Prathibaa, Dr. K.Gunasekaran., Planning of Bus Stops for Safe and Efficient Passenger Boarding and Alighting, International Journal of Engineering Research \& Technology (IJERT) ISSN: 2278-0181, (5), pp. 296-304. (2016).

6. Salvatore Cafiso, Alessandro Di Graziano, Giuseppina Pappalardo, Road safety issues for bus transport management, Transport Research Arena - Europe. (48), pp. 22512261. (2012).

7. Fabio Porcu, Alessandro Olivo, Giulio Maternini, Benedetto Barabino., Evaluating bus accident risks in public transport, Transportation Research Procedia, (45), pp. 443-450.( 2020).

8. Syyed Adnan Raheel Shah, Naveed Ahmad, Yongjun Shen, Ali Pirdavani, Muhammad Aamir Basheer, and Tom Brijs. Road Safety Risk Assessment: An Analysis of Transport Policy and Management for Low-, Middle-, and High-Income Asian Countries. Sustainability, (10). pp. 1-30. (2018).

9. I Farida., Public transport service relating to safety, IOP Conf. Series: Materials Science and Engineering, pp 434 (2018).

10. Huaguo, Zhou, Pey-Sung Lin, Joan Shen. An Unconventional Design for Bus U-Turns at Signalized Intersections. Journal of Public Transportation, (11), pp. 89-103. (2008).

11. Anna Izabel J. Tostes, Fatima de L. P. Duarte-Figueiredo, Renato Assuncao, Juliana Salles, Antonio A. F. Loureiro. From Data to Knowledge: City-wide Traffic Flows Analysis and Prediction Using Bing Maps, Conference: Proceedings of the 2nd ACM SIGKDD International Workshop on Urban Computing, (2013).

12. Amit Prasad Timalsena, Anil Marsani, Hemant Tiwari. Impact of Traffic Bottleneck on Urban Road: A Case Study of Maitighar-Tinkune Road Section. Proceedings of IOE Graduate Conference,. pp. 623-628. (2017).

13. Aleksandra Koźlak, Dagmara Wach. Causes of traffic congestion in urban areas. Case of Poland. SHS Web of Conferences 57, 01019. pp.1-9.(2018).

14. Mrs.Swati Chandurkar, Sneha Mugade, Sanjana Sinha, Megharani Misal, Pooja Borekar, Implementation of Real Time Bus Monitoring and Passenger Information System, International Journal of Scientific and Research Publications, 5, (3),.pp. 15.(2013).

15. Seiya Matsuoka, Traffic management project in Phnom Penh. IATSS Research 42. pp.180-189. (2018).

16. Alimukhamedov Sh.P., Abdullaev B.I, Improving the method of assessing the safety of public transport. Scientific Journal of Mechanics and Technology, (1), pp. 55-62. (2020). 
17. Kasatkin F.P. Organization of transport services and safety of the transport process, study guide F.L. Kasatkin, Sh. A. Amirseiids; Vladimir State University named after A.G. and N.G. Stoletovs. - Vladimir: Publishing house of VISU, p 264. (2014).

18. Litvin V.V. Development of a methodology for a comprehensive assessment of the quality of transport services for passengers on urban bus routes in medium and large cities,

19. Silyanov V.V, and other safety of road traffic. Reference road builder encyclopedia (SED) .T. VIII / Silyanov V.V., V.A.Aksenov, B. B. Anokhin, V. V. Chvanov, I. F. Zhivopistsev, A. P. Shevyakov and others; / Ed. Doctor of Technical Sciences V.V. Silyanov. - M .: FGUP "INFORMAVTODOR", p 751. (2008).

20. IRG (Industry road guidance document) 218.4.005-2010, Recommendations for traffic safety on highways, Federal Road Agency (RosAvtoDor), Moscow (2011) 